\title{
Laws of Resistance in Transitional Pipe Flows
}

\author{
Rory T. Cerbus, Chien-chia Liu, Gustavo Gioia, and Pinaki Chakraborty* \\ Okinawa Institute of Science and Technology Graduate University, Onna-son, Okinawa 904-0495, Japan
}

(Received 24 July 2017; published 31 January 2018)

\begin{abstract}
As everyone knows who has opened a kitchen faucet, pipe flow is laminar at low flow velocities and turbulent at high flow velocities. At intermediate velocities, there is a transition wherein plugs of laminar flow alternate along the pipe with "flashes" of a type of fluctuating, nonlaminar flow that remains poorly understood. In the 19th century, Osborne Reynolds sought to connect these states of flow with quantitative "laws of resistance," whereby the fluid friction is determined as a function of the Reynolds number. While he succeeded for laminar and turbulent flows, the laws for transitional flows eluded him and remain unknown to this day. By properly distinguishing between laminar plugs and flashes in the transitional regime, we show experimentally and numerically that the law of resistance for laminar plugs corresponds to the laminar law and the law of resistance for flashes is identical to that of turbulence.
\end{abstract}

DOI: 10.1103/PhysRevLett.120.054502

In 1883, Osborne Reynolds [1] carried out a series of experiments to test the theoretical argument, rooted in the concept of dynamical similarity, that the character of a pipe flow should be determined by the Reynolds number $\operatorname{Re} \equiv U D / \nu$, where $U$ is the mean velocity of the flow, $D$ is the diameter of the pipe, and $\nu$ is the kinematic viscosity of the fluid. At low Re, Reynolds observed a state of flow, known as laminar, in which "the elements of the fluid follow one another along lines of motion which lead in the most direct manner to their destination" [1]. At high Re, Reynolds observed another state of flow, known as turbulent, in which the elements of fluid "eddy about in sinuous paths the most indirect possible" [1]. At intermediate values of Re, Reynolds found a transitional regime in which the flow was spatially heterogeneous, with plugs of steady, presumably laminar, flow alternating with flashes (Reynolds's term) of fluctuating, eddying flow.

Since Reynolds's time, much has been learned about the transitional regime. It is now known that there are two types of flash [2-6]: "puffs" and "slugs." The puffs appear first, at the onset of the transitional regime, which is triggered by finite-amplitude perturbations [5]. Shaped like downstream-pointing arrowheads [2,5], the puffs are $\approx 20 D$ long and travel downstream at a speed [5] of $\approx 0.9 U$. A puff may decay and disappear, or it may split into two or three puffs separated by intervening laminar plugs [7]. With further increase in Re, the puffs are replaced by slugs [8] $(\operatorname{Re} \gtrsim 2250)$. With a head faster than $U$ and a tail that is slower, the slugs, unlike the puffs, spread as they travel

Published by the American Physical Society under the terms of the Creative Commons Attribution 4.0 International license. Further distribution of this work must maintain attribution to the author(s) and the published article's title, journal citation, and DOI. downstream and are therefore capable of crowding out the laminar plugs. As Re increases further, the flow turns turbulent. The stark contrast between puffs and slugs and turbulence suggests that each constitutes a distinct state of flow.

Despite the noteworthy advances in our knowledge, including the recent elucidation of the link between transition in shear flows and the directed percolation universality class $[6,9,10]$, a major goal of Reynolds's inquiry [1] still eludes us. Seeking to classify the states of flow as laminar, turbulent, or transitional, Reynolds investigated laws of resistance that express the fluid friction $f$ (a unitless measure of pressure drop per unit length of pipe) as a function of $\operatorname{Re}$ (see, e.g., Fig. 1). (By definition, $f \equiv\left[D \Delta P / \Delta L /\left(\rho U^{2} / 2\right)\right]$, where $\Delta P / \Delta L$ is the pressure drop per unit length of pipe and $\rho$ is the density of the fluid; see Supplemental Material [11].) For laminar flow, Reynolds [1] found that $f=f_{\text {lam }}(\operatorname{Re}) \equiv 64 / \mathrm{Re}$, which is known as the Hagen-Poiseuille law, an exact mathematical result derivable from the equations of motion for a flow dominated by viscosity. Fluctuations have a marked effect on $f$, and for turbulent flow, Reynolds [1] found that $f=f_{\text {turb }}(\operatorname{Re}) \equiv 0.3164 \mathrm{Re}^{-1 / 4}$, an empirical result known as the Blasius law $[18,19]$. The Blasius law has not yet been derived from the equations of motion, but it has recently been shown that the scaling $f_{\text {turb }}(\mathrm{Re}) \propto \mathrm{Re}^{-1 / 4}$ can be derived from Kolmogorov's phenomenological theory of turbulence [20,21], which furnishes a direct link between this law of resistance and the internal structure of the flow. Finally, for transitional flow, Reynolds found that the relation between $f$ and $\mathrm{Re}$ "was either indefinite or very complex" [1], and to date the laws for the transitional regime-the focus of this Letter-remain unknown [5]. To illustrate the problems in seeking these laws, we begin with a discussion of our experiments. 


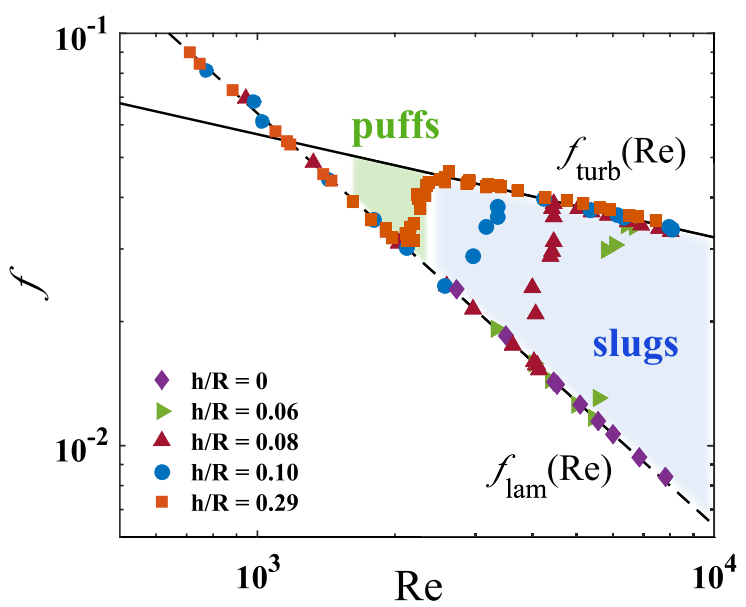

FIG. 1. A typical $f$ vs Re state diagram. The position of a data point $(f, \mathrm{Re})$ can be used to classify the state of the flow: laminar flow corresponds to $f_{\text {lam }}(\mathrm{Re})$ (dashed line), turbulent flow corresponds to $f_{\text {turb }}(\mathrm{Re})$ (solid line), and transitional flow corresponds to the region between $f_{\text {lam }}(\mathrm{Re})$ and $f_{\text {turb }}(\mathrm{Re})$ (puffs and slugs occupy two distinct regions, marked in green and blue, respectively.) The data points are from experiments where we perturb the flow using an obstacle (iris) of size $h$ along the periphery of the pipe of radius $R$. Each set of data points corresponds to a fixed value of $h / R$. For $h / R=0$ (no obstacle), the flow is laminar for all Re tested. With increase in $h / R$, the onset of the transitional regime shifts to lower Re.

We carry out measurements of $f$ in a 20-m-long, smooth, cylindrical glass pipe of $D=2.5 \mathrm{~cm} \pm 10 \mu \mathrm{m}$. The fluid is water. Driven by gravity, the flow remains laminar up to the highest $\operatorname{Re}$ tested $(\operatorname{Re} \approx 8300)$. To make flashes, we perturb the flow using an obstacle (iris) or a pair of syringe pumps. We compute data points $(f, \mathrm{Re})$ by measuring, at discrete times $t$, the mean velocity $U$ over the cross section and the pressure drop $\Delta P$ over a length span $\Delta L=202 D$. The time series $\Delta P(t)$ and $U(t)$ yield $f(t)$ and $\operatorname{Re}(t)$, which we average over a long time $(>4000 D / U)$ to obtain a single data point $(f$, $\mathrm{Re})$. (See Supplemental Material for further discussion [11]).

In Fig. 1, a log-log plot of $f$ vs Re, we plot five representative sets of data points. We see data points that fall on $f_{\text {lam }}(\mathrm{Re})$ and correspond to laminar flows, data points that fall on $f_{\text {turb }}(\mathrm{Re})$ and correspond to turbulent flows, and data points that fall between $f_{\text {lam }}(\mathrm{Re})$ and $f_{\text {turb }}(\mathrm{Re})$ [22] and correspond to transitional flows. In the transitional regime, the relation between $f$ and Re is not unique and there appear to be no discernible laws. Standard engineering plots of $f$ vs Re-called Moody diagrams $[19,23]$ - show the transitional regime blank or hashed out, symbolizing our ignorance. Recent research on $f$ for exact coherent structures (ECSs) further complicates the problem [24]. (Thought to be the building blocks of puffs and slugs, ECSs are special transient solutions of the Navier-Stokes equations [4].) Whereas experiments show $f_{\text {turb }}(\mathrm{Re})$ to be an upper bound for $f$ in the transitional regime, $f$ for some ECSs manifestly overshoot $f_{\text {turb }}(\operatorname{Re})$, a puzzle to which we return later.

To seek clues regarding $f$ in the transitional regime, we first consider transitional flows with slugs. From Fig. 1, we pick one set of data points and replot them in Fig. 2(a). In Fig. 2(b), we show the time series $f(t)$ and the attendant probability distribution function $p(f)$ for a representative laminar data point (marked $L$ ), for a representative transitional data point (marked $S$ ), and for a representative turbulent data point (marked $T$ ). For point $L$ and for point (a)

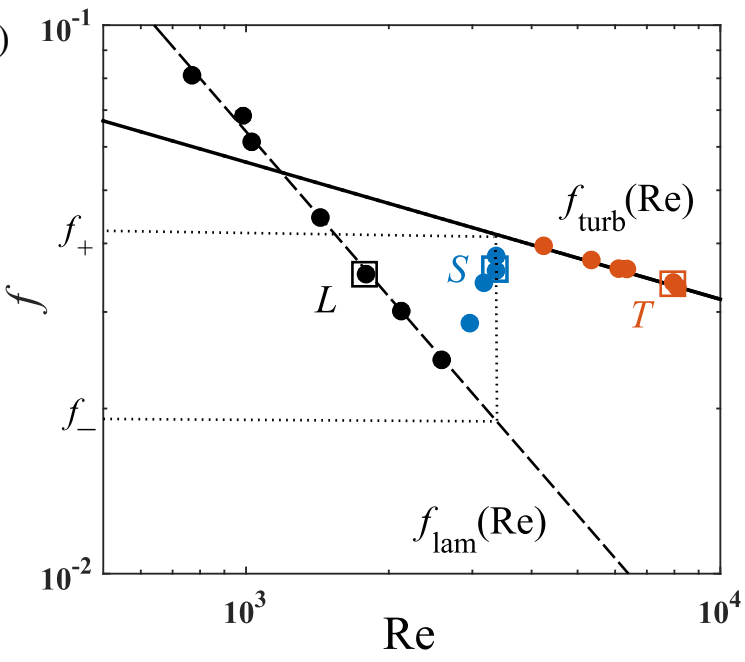

(b)
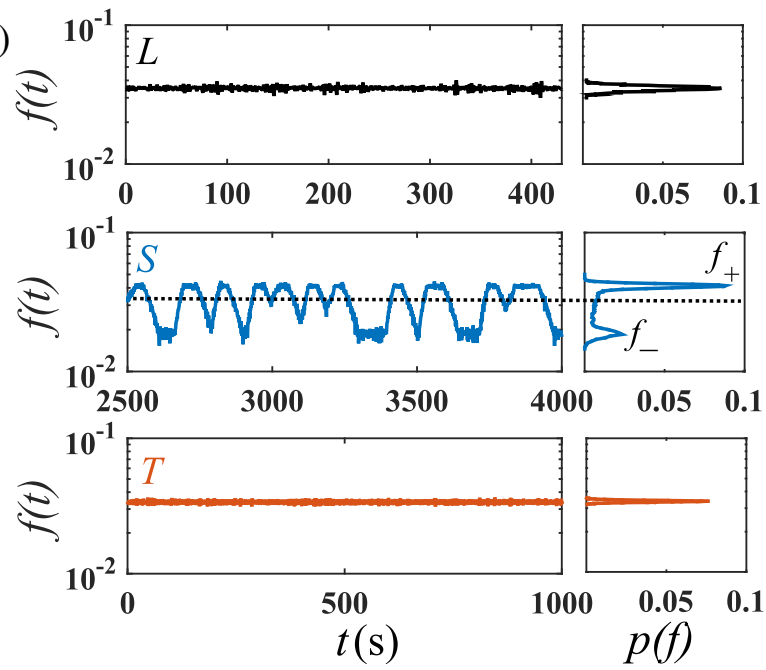

FIG. 2. A closer look at $f$ for transitional flows with slugs. (a) Data points ( $f, \operatorname{Re}$ ) corresponding to laminar flows (shown in black), transitional flows (shown in blue), and turbulent flows (shown in orange); from Fig. $1(h / R=0.10)$. (b) The time series $f(t)$ and the attendant probability distribution function $p(f)$ for the data points marked $L, S$, and $T$ in (a). Note that, for the transitional data point $S$, $f(t)$ swings around the dotted line [the long-time average of $f(t)$ ], between two distinct values $f_{-}$and $f_{+}$, where $f_{-}=f_{\text {lam }}(\operatorname{Re})$ and $f_{+}=f_{\text {turb }}(\operatorname{Re})$, as indicated in (a). 
(a)
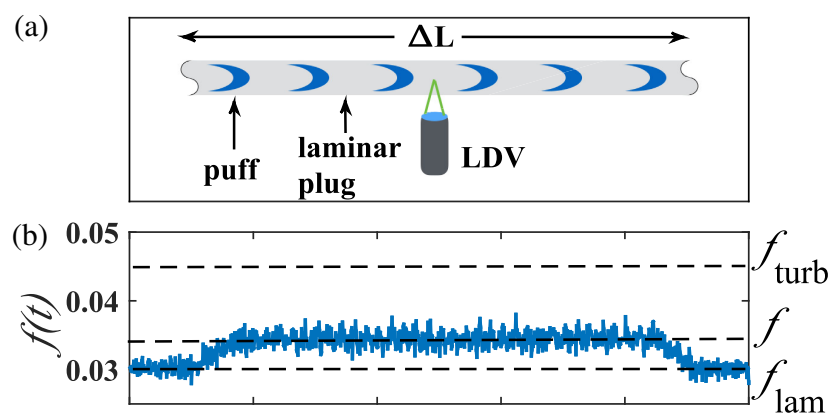

(c)

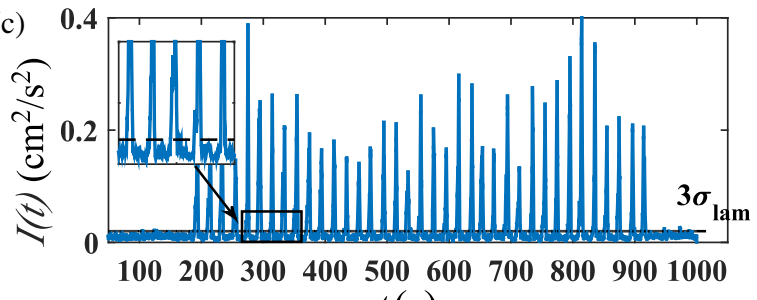

$t(\mathrm{~s})$

FIG. 3. Computing $f$ for puffs, $f_{\text {puffs }}$ (a) Schematic of the pipe section where we measure the pressure drop for a train of puffs. The laser Doppler velocimeter (LDV) stationed at the middle of this section measures the time series of the radial velocity at the centerline $v(t)$. We also simultaneously measure the time series of pressure drop $\Delta P(t)$ and mean velocity $U(t)$. (b) Sample time series $f(t)$ for a train of about 30 puffs. We compute $f(t)$ using $\Delta P(t)$ and $U(t) . f$ is the time-averaged $f(t)$ for the train of puffs. For reference, we mark $f_{\text {lam }}$ and $f_{\text {turb }}$ at the flow Re. (c) Attendant to (b), simultaneous measurements of the indicator function $I(t)=\left\langle v(t)^{2}\right\rangle_{\Delta T}$. We mark the threshold $\delta=3 \sigma_{\text {lam }}$. (Inset) Enlarged view. The puffs correspond to $I(t) \geq \delta$.

$T, p(f)$ has a single peak, the value of which is the same as the long-time average of $f(t)$, which we have denoted by $f$. By contrast, for the transitional data point $S, p(f)$ has two peaks and $f(t)$ swings [25] between the peak values, marked $f_{-}$and $f_{+}$in Fig. 2, spending little time at the long-time average of $f(t)$, which includes contributions from both laminar plugs and slugs. Here, $f_{-}$is the friction for laminar plugs (that is, the unitless pressure drop per unit length of laminar plug) and $f_{+}$is the friction for slugs (that is, the unitless pressure drop per unit length of slug). But there is more: it turns out that $f_{-}=f_{\text {lam }}(\operatorname{Re})$ and $f_{+}=$ $f_{\text {turb }}(\mathrm{Re})$ [as indicated in Fig. 2(a)] - and not just for data point $S$, but for all transitional data points in Fig. 2(a).

We now turn to transitional flows with puffs. Unlike slugs, puffs are short $(\approx 20 D$ long $)$ as compared to the length span $\Delta L=202 D$, where we measure the pressure drop $\Delta P$, and the technique we have used to measure $f$ for slugs cannot be used for puffs. To measure $f$ for puffs, $f_{\text {puffs }}$, we create a train of puffs $[22,26]$ such that, at any given time, about 6-7 puffs fit within $\Delta L$ [Fig. 3(a)]. We then measure the time series $f(t)$, which we average over the train of puffs to obtain $f$ [Fig. 3(b)]. Now, this $f$ includes contributions from both puffs and laminar plugs. Employing the lever rule for binary mixtures [27], we write [28]

$$
f=\gamma f_{\text {puffs }}+(1-\gamma) f_{\text {lam }}
$$

where the intermittency $\gamma$ is the fraction of the flow corresponding to puffs: $0 \leq \gamma \leq 1$. Once we deduce $\gamma$ (discussed next), we use the experimentally determined $f_{\text {lam }}$ (corresponding to the laminar flow before and after the train of puffs) and solve for $f_{\text {puffs }}$.

To determine $\gamma$, we measure the time series of the radial velocity at the centerline $v(t)$ [Fig. 3(a)] and identify puffs to be the regions where the indicator function $I(t)$, which we define as the moving average of $v^{2}(t)$ over a time window $\Delta T=5 D / U,\left\langle v^{2}(t)\right\rangle_{\Delta T}$, exceeds a given threshold $\delta$ [29]. Similar to Ref. [10], we set $\delta=3 \sigma_{\text {lam }}$, where $\sigma_{\text {lam }}$, the standard deviation of $\left\langle v^{2}(t)\right\rangle_{\Delta T}$ for a laminar flow at the same Re as the train of puffs, is a measure of the instrumental and experimental noise. The puffs correspond to $I(t) \geq \delta$ [Fig. 3(c)]. The fraction of $t$ where this inequality holds yields $\gamma$, which, via Eq. (1), yields $f_{\text {puffs }}$ (see Supplemental Material [11]). Note that the same procedure can be applied to transitional flows with slugs, as an alternative to the simpler procedure that we used to obtain the results as in Fig. 2(b) the results differ by $<1.5 \%$.

All of our experimental results for $f$ rely on measuring the global pressure drop over the length span $\Delta L=202 D$. To complement these results, we conduct direct numerical simulations [30], wherein we compute $f$ using the local $f(x)$, where $x$ is the position along the pipe axis (see Supplemental Material [11]). From the simulated flow fields, we calculate $f(x)=8 \tau(x) / \rho U(x)^{2}$, where $\tau$ is the shear stress at the wall. To separate flashes (either slugs or puffs, as the case might be) from laminar plugs, we define an indicator function $I(x)$ as the cross-sectional average of the squared in-plane velocity fluctuations (radial and azimuthal) [31]. Now, without needing to resort to Eq. (1), we directly compute $f$ for flashes as the average of $f(x)$ corresponding to $I(x) \geq \delta$ and $f$ for laminar plugs as the average of $f(x)$ corresponding to $I(x)<\delta$. We are now ready to collate the results.

In Fig. 4(a), a log-log plot of $f$ vs Re, we show all the transitional data points from our experiments and simulations. As expected, the transitional data points corresponding to puffs and the transitional data points corresponding to slugs appear dispersed as two clouds of data points, with no hint of any laws of resistance. For each of the transitional data points in Fig. 4(a), we compute $f$ for flashes (slugs as well as puffs) and $f$ for laminar plugs, and plot the outcome in Fig. 4(b) to arrive at our main results: for all transitional flows, $f$ for laminar plugs equals $f_{\text {lam }}(\mathrm{Re})$ and $f$ for flashes equals $f_{\text {turb }}(\mathrm{Re})$, irrespective of the type of flash. That is, the very same laws of resistance Reynolds found for laminar flows and turbulent flows directly extend to laminar plugs and flashes, respectively [32]. Because the laws of resistance are a conventional diagnostic for the state of flow, we conclude that laminar plugs are indeed laminar and, more importantly, that puffs and slugs are turbulent, even though 

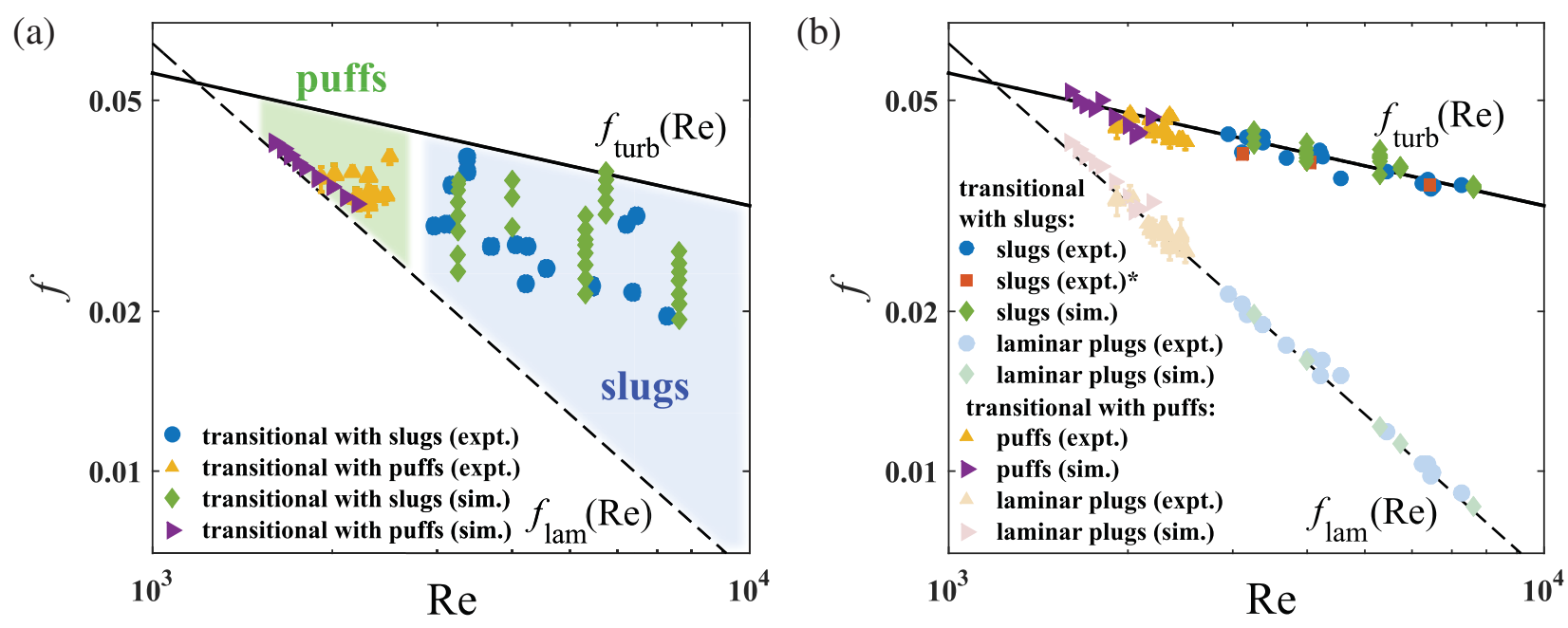

FIG. 4. Experimental and numerical measurement of $f$ vs $\operatorname{Re}$ for transitional flows. (a) Data points $(f$, Re) corresponding to transitional flows with slugs and transitional flows with puffs. For each data point, $f$ includes a contribution from laminar plugs and a contribution from flashes. By disentangling these contributions, we obtain $f$ for laminar plugs and $f$ for flashes and plot the disentangled $f$ vs Re in (b). In all cases, $f$ for laminar plugs falls on $f_{\text {lam }}(\operatorname{Re})$ and $f$ for flashes falls on $f_{\text {turb }}(\operatorname{Re})$. For most data points [(a),(b)], the error bars in $f$ and the error bars in Re are smaller than the size of the data points. The exceptions are the experimental data of $f$ for transitional flows with puffs; the vertical error bars indicate uncertainties in $f$ (see Supplemental Material [11]). (b) For the data points marked with $*$, we compute $f$ for slugs using the same procedure that we use to compute $f$ for puffs.

puffs and slugs and turbulence outwardly appear to be three distinct states of flow.

Based on our results, we can write $f$ for any transitional data point using the lever rule as $\gamma f_{\text {turb }}(\mathrm{Re})+$ $(1-\gamma) f_{\text {lam }}(\mathrm{Re})$. This does not, however, fully resolve Reynolds's original quandary. To determine the value of $f$, we still need to know the corresponding $\gamma$, but at present there is no theory relating $\gamma$ to Re. That said, the framework of directed percolation might provide a starting point [33].

Our results also hint at an analogy between the transitional regime and the phenomenon of shear banding in complex fluids $[34,35]$. For a range of shear rates, sheared flow of complex fluids becomes spatially heterogeneous, forming a weakly sheared band and a highly sheared band. Here, the total shear rate is governed by a lever rule between shear rates of the weakly sheared band and the highly sheared band, analogous to $f$ for laminar plugs and flashes, respectively. The attendant shear stress remains nearly constant for the range of shear rates, analogous to the limited variation of Re as $f$ traverses between $f_{\text {lam }}(\operatorname{Re})$ and $f_{\text {turb }}(\operatorname{Re})$ (see Fig. 1). The analogy between transition and shear banding may lead to a useful exchange between these two seemingly unrelated fields.

Last, we return to the puzzle of $f$ for ECSs [24]. Using the framework of ECSs, $f$ for flashes can be computed as a weighted average of $f$ for individual ECSs, where the weight is the fraction of time the flow resides in the phasespace region corresponding to the ECS. Since the values of $f$ for the individual ECSs are unlikely to be all identical, the individual $f$ values, constrained by our result $[f$ for flashes equals $f_{\text {turb }}(\mathrm{Re})$ ] must necessarily go above and below the weighted average $f_{\text {turb }}(\operatorname{Re})$. Over- and undershoots are thus but expected. In addition, we note that $f$ for a complete set of ECSs must satisfy $f_{\text {turb }}(\mathrm{Re})$. This provides a necessary condition for the completeness of the set, akin to the sum rules in quantum mechanics [36].

A staple of undergraduate curricula, the $f$ vs Re diagram is familiar to every fluid mechanist. And yet, closely reexamining this 130 -year-old diagram continues to yield fresh insights.

We thank Professor Jun Sakakibara (Meiji University) and Mr. Makino (Ni-gata company) for help with the experimental setup, Dr. Ashley Willis (University of Sheffield) for making OPENPIPEFLOW freely available, and an anonymous referee for suggesting the analogy with shear banding. This work was supported by the Okinawa Institute of Science and Technology Graduate University. R. T.C. gratefully acknowledges the support of JSPS (KAKENHI Grant No. 17K14594).

* Corresponding author. pinaki@oist.jp

[1] O. Reynolds, Phil. Trans. R. Soc. London 174, 935 (1883).

[2] I. Wygnanski and F. Champagne, J. Fluid Mech. 59, 281 (1973).

[3] I. Wygnanski, M. Sokolov, and D. Friedman, J. Fluid Mech. 69, 283 (1975).

[4] B. Eckhardt, T. M. Schneider, B. Hof, and J. Westerweel, Annu. Rev. Fluid Mech. 39, 447 (2007).

[5] T. Mullin, Annu. Rev. Fluid Mech. 43, 1 (2011).

[6] D. Barkley, J. Fluid Mech. 803, P1 (2016). 
[7] K. Avila, D. Moxey, A. de Lozar, M. Avila, D. Barkley, and B. Hof, Science 333, 192 (2011).

[8] D. Barkley, B. Song, V. Mukund, G. Lemoult, M. Avila, and B. Hof, Nature (London) 526, 550 (2015).

[9] Y. Pomeau, Nat. Phys. 12, 198 (2016); H.-Y. Shih, T.-L. Hsieh, and N. Goldenfeld, Nat. Phys. 12, 245 (2016); G. Lemoult, L. Shi, K. Avila, S. V. Jalikop, M. Avila, and B. Hof, Nat. Phys. 12, 254 (2016).

[10] M. Sano and K. Tamai, Nat. Phys. 12, 249 (2016).

[11] See Supplemental Material at http://link.aps.org/ supplemental/10.1103/PhysRevLett.120.054502 for additional discussion, which includes Refs. [12-17].

[12] J. Den Toonder and F. Nieuwstadt, Phys. Fluids 9, 3398 (1997).

[13] S. G. Huisman, D. P. van Gils, and C. Sun, Eur. J. Mech. B 36, 115 (2012).

[14] M. Nishi, B. Ünsal, F. Durst, and G. Biswas, J. Fluid Mech. 614, 425 (2008).

[15] A. P. Willis and R. R. Kerswell, J. Fluid Mech. 619, 213 (2009).

[16] J. Eggels, F. Unger, M. Weiss, J. Westerweel, R. Adrian, R. Friedrich, and F. Nieuwstadt, J. Fluid Mech. 268, 175 (1994).

[17] X. Wu and P. Moin, J. Fluid Mech. 608, 81 (2008).

[18] The Blasius law provides an excellent fit to experimental data on turbulent flow for $\operatorname{Re}<10^{5}$.

[19] P. Steen and W. Brutsaert, Annu. Rev. Fluid Mech. 49, 575 (2017).

[20] G. Gioia and F. Bombardelli, Phys. Rev. Lett. 88, 014501 (2001).

[21] G. Gioia and P. Chakraborty, Phys. Rev. Lett. 96, 044502 (2006).

[22] D. Samanta, A. De Lozar, and B. Hof, J. Fluid Mech. 681, 193 (2011).

[23] L. F. Moody, Trans. Am. Soc. Mech. Eng. 66, 671 (1944).

[24] H. Wedin and R. Kerswell, J. Fluid Mech. 508, 333 (2004); F. Mellibovsky, A. Meseguer, T. M. Schneider, and B. Eckhardt, Phys. Rev. Lett. 103, 054502 (2009).

[25] F. Durst and B. Ünsal, J. Fluid Mech. 560, 449 (2006).

[26] B. Hof, A. De Lozar, M. Avila, X. Tu, and T. M. Schneider, Science 327, 1491 (2010).
[27] C. Kittel and H. Kroemer, Thermal Physics, 2nd ed. (W. H. Freeman, San Francisco, 1980).

[28] See [37] for a similar approach in transitional boundarylayer flows.

[29] Our approach is similar to previous work [9,10,31,38], which defined $I(t)$ as the cross-sectional average of the squared inplane velocity (or vorticity) fluctuations. For our single-point measurement, an analogous measure is the moving average of $v^{2}(t)$ over a time window $\Delta T$. The moving average, while seemingly unsophisticated, is optimal for reducing noise and enhancing step response [39], allowing us to cleanly identify puffs.

[30] A. P. Willis, SoftwareX 6, 124 (2017).

[31] D. Moxey and D. Barkley, Proc. Natl. Acad. Sci. U.S.A. 107, 8091 (2010).

[32] These results validate the primary assumptions invoked in [40].

[33] If directed percolation governs $\gamma$, we can write $\gamma \propto$ $\left(\operatorname{Re}-\operatorname{Re}_{c}\right)^{\beta}$, where $\operatorname{Re}_{c}$ is the $\operatorname{Re}$ corresponding to the onset of transition and $\beta$ is a critical exponent whose value is 0.2769 for one-dimensional directed percolation.

[34] J.-B. Salmon, A. Colin, S. Manneville, and F. Molino, Phys. Rev. Lett. 90, 228303 (2003).

[35] T. Divoux, M. A. Fardin, S. Manneville, and S. Lerouge, Annu. Rev. Fluid Mech. 48, 81 (2016).

[36] Sum rules in quantum mechanics, a famous example being the Thomas-Reiche-Kuhn sum rule for oscillators, are exact results for the sum of transition probabilities between energy states. These model-independent rules played a crucial role in the historical development of quantum mechanics [41].

[37] K. P. Nolan and T. A. Zaki, J. Fluid Mech. 728, 306 (2013).

[38] M. Avila and B. Hof, Phys. Rev. E 87, 063012 (2013).

[39] S. Smith, Digital Signal Processing: A Practical Guide for Engineers and Scientists (Newnes, Burlington, MA, 2013).

[40] A. C. Fowler and P. Howell, SIAM J. Appl. Math. 63, 1184 (2003).

[41] J. J. Sakurai and J. J. Napolitano, Modern Quantum Mechanics (Pearson, London, 2014). 